Ass.-Prof. Dr. Lamyaa Abdelmohsen Osman Ali Ziko

\title{
Literatur im Rahmen akademischer Vorgaben der Germanistik-Ausbildung: Ästhetische Impulse und didaktische Outcomes ${ }^{1}$ Ass.-Prof. Dr. Lamyaa Abdelmohsen Osman Ali Ziko \\ Abteilung für deutsche Sprache und Literatur Philosophische Fakultät, Menoufia Universität
}

\section{Abstract}

Die Behandlung literarischer Werke und die Vermittlung von literaturwissenschaftlichen Inhalten gehören $\mathrm{zu}$ den Bausteinen einer germanistischen Ausbildung und sind essentielle curriculare akademische Vorgaben. In der internationalen Germanistik und mit der Entstehung des Fachbereichs $\mathrm{DaF}$ haben sich theoretische Ansätze in der fremdsprachlichen Literaturdidaktik entwickelt, deren Ideen in unterschiedliche Lehrmaterialien für den fremdsprachlichen Literaturunterricht Eingang gefunden haben. Gleichzeitig sind zur Systematisierung des Fremdsprachenerwerbs Referenzrahmen auf europäischer und regionaler Ebene entstanden - wie der GER für den Fremdsprachenerwerb oder die ägyptischen Referenzstandards (NARS) für die universitäre Fremdsprachenphilologie -, die ein gemeinsames Fundament für sprachliche Fertigkeiten anstreben und damit internationale Gültigkeit zu finden beabsichtigen.

Der Beitrag stellt vor diesem Hintergrund den Stellenwert von Literatur und Literaturwissenschaft in den genannten Referenzrahmen vor und untersucht anschließend, inwieweit moderene Ansätze am Beispiel des Materialbandes Literatur Lesen Lernen von Dobstadt/Riedner (2017) den Anforderungen und Standards dieser Referenzrahmen gerecht werden. Es wird seine

1 Der Beitrag beruht auf dem Vortrag „Literatur im Rahmen akademischer Vorgaben der Germanisti-Ausbildung: Vermittlung von Sprache, Kultur, Wissen und berufsbezogenen Kompetenzen. Ist das machbar?", der während der vom EZ-DAAF organisierten Konferenz „Deutsch und Arabisch im internationalen Kontext. 10 Jahre EZ-DAAF: Bilanz und Perspektiven“ vom 08. - 10.10.2018 gehalten wurde.

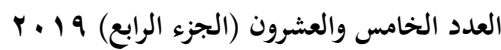

مجلة كلية التربية- جامعة عين شمس 
Literatur im Rahmen akademischer Vorgaben der GermanistikAusbildung: Ästhetische Impulse und didaktische Outcomes

konzeptionelle Fundierung, die symbolische Komptetenz von Kramsch (2011), aufgezeigt, deren Realisierung im Materialband aufgehellt und schließlich seine Einsetzbarkeit im universitären literaturwissenschaftlichen Kontext ausgewertet werden.

Schlüsselwörter: Interkulturalität, Interkulturelle Germanistik, DaF, Fremdsprachliche Literaturdidaktik, Symbolische Kompetenz, Rezeptionsästhetik 


\section{الأدب فى إطار المتطلبات الأكاديمية لتعليم اللغة الألمانية وآدابها: المحفزات الجمالية والمخرجات التربوية$$
\text { أ.م.د. لمياء عبدالمحسن عثمان على زيكو }
$$$$
\text { قسم اللغة الألمانية وآدابها }
$$$$
\text { كلية الآداب، جامعة المنوفية }
$$

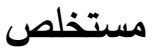

إن معالجة الأدب من خلال الأعمال الأدبية وموضوعات علم الأدب من لبنات دراسة علوم اللغة الألمانية وآدابها فى الجامعات المصرية، كما أنها من المتطلبات الأكاديمية الرئيسية فى لوائح

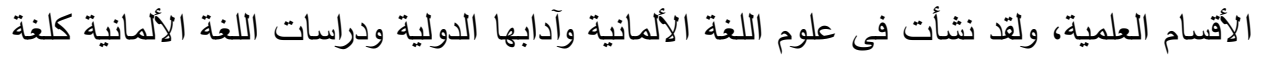

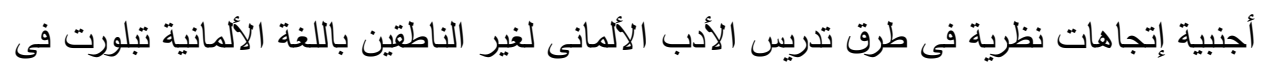

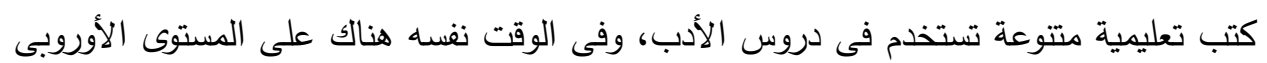

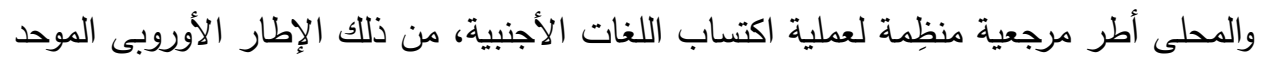

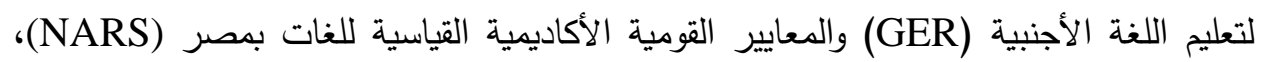
ويسعى كل منهما إلى وضع أساس موحد لاكتساب اللغة وإلى الاعتراف على المستوى الدولى.

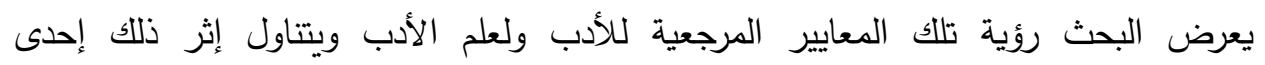

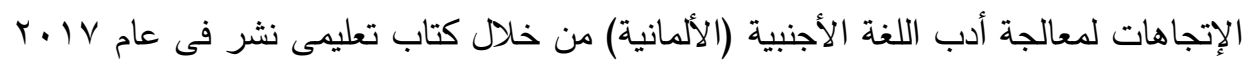

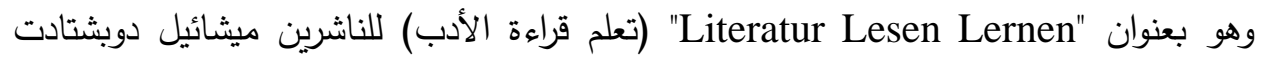
وريناته ريدنر (Dobstadt/Riedner)، من حيث المنهج المتبع والموضوعات المطروحة فيه؛ يتبع الكتاب منهج المهارة الرمزية لإحدى علماء الجرمانيات كلير كرامش (Kramsch) (Y (11)، كما يقوم البحث بتحليل مدى تحقيق متطلبات المعايير القياسية الأوروربة والمصرية

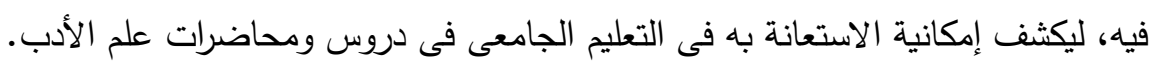

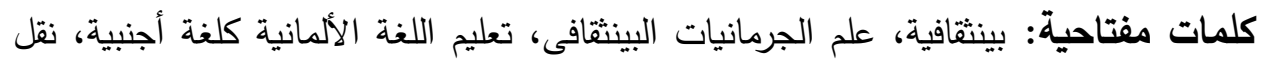
أدب اللغة الأجنبية، مهارة رمزية، نظرية التلقى الجمالية. 


\section{Literatur im Rahmen akademischer Vorgaben der Germanistik-Ausbildung: Ästhetische Impulse und didaktische Outcomes ${ }^{2}$ Ass.-Prof. Dr. Lamyaa Abdelmohsen Osman Ali Ziko \\ Abteilung für deutsche Sprache und Literatur \\ Philosophische Fakultät, Menoufia Universität}

\section{Einleitung}

Der Sinn der Vermittlung von Literaturwissenschaft und der wissenschaftlichen Auseinandersetzung mit literarischen Texten innerhalb des Germanistik-Studiums in Ägypten wird nicht nur von Studierenden in Frage gestellt. Eine nähere Beobachtung des ägyptischen Arbeitsmarkts legt die Vermutung nahe, dass Kenntnis über Literatur und Literaturwissenschaft als berufsbezogenes Erfordernis bei weitem keine Rolle spielt. Vielmehr sind zunächst kommunikative Kompetenzen gefordert, die zu einer effizienten Aufnahme und Vermittlung von Informationen führen sollen, aber auch soziale Fähigkeiten und Handlungskompetenzen, die beim Umgang mit dem deutschsprachigen Gesprächspartner notwendig sind. Der deutschsprechende Arbeitnehmer muss demzufolge eigens in der Lage sein, einen berufsspezifischen Kommunikationsprozess in der deutschen Sprache erfolgreich und professionell auszuführen. Die Kenntnis über Literatur und über ihre Wissenschaft ist in Berufsfeldern wie Kundendienst, Tourismus, Medien oder administrativen Tätigkeiten in deutschsprachigen Institutionen irrelevant. Auch im Lehrerberuf scheint eine sprachwissenschaftliche Ausbildung sinnvoller $\mathrm{zu}$ sein als die Aneignung von literaturwissenschaftlichen Inhalten. Wie Literatur in diesen Arbeitsfeldern präsent und/oder einsetzbar ist, ist

2 Der Beitrag beruht auf dem Vortrag „Literatur im Rahmen akademischer Vorgaben der Germanisti-Ausbildung: Vermittlung von Sprache, Kultur, Wissen und berufsbezogenen Kompetenzen. Ist das machbar?", der während der vom EZ-DAAF organisierten Konferenz „Deutsch und Arabisch im internationalen Kontext. 10 Jahre EZ-DAAF: Bilanz und Perspektiven“ vom 08. - 10.10.2018 gehalten wurde.

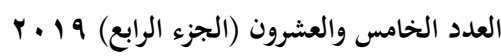

مجلة كلية التربية- جامعة عين شمس 
Ass.-Prof. Dr. Lamyaa Abdelmohsen Osman Ali Ziko

dementsprechend den Studierenden wie auch Arbeitnehmern nicht eindeutig. Literatur und Literaturwissenschaft innerhalb der germanistischen Ausbildung in Ägypten könnte in dieser Hinsicht als zweckloser Teil des Studiums erscheinen. Denn es führt zu keiner spezifischen Beschäftigung im aktuellen Arbeitsmarkt Ausnahmen ausgeschlossen.

Einer derartigen Vorstellung muss allerdings entgegnet werden, dass auch ein internationales Germanistik-Studium ohne Literatur und Literaturwissenschaft nicht als solches zu betrachten wäre. Den Interessierten an das Erlernen der deutschen Sprache wäre zu empfehlen, ein anderes Studium $\mathrm{zu}$ wählen und nebenbei Deutschkurse zu belegen. Tatsächlich braucht der Arbeitsmarkt nicht nur kompetente Sprecher, sondern auch Kulturversteher, die vor allem für die kulturellen Hintergründe der fremdsprachigen Gesprächspartner sensibilisiert sind. Kultur ist vor allem durch literarische Werke wirkungsvoll $\mathrm{zu}$ vermitteln als allein durch linguistische Spracharbeit, faktenorientierten landeskundlichen Wissenserwerb oder kommunikativ orientierten Fremdsprachenunterricht. Ihre Weltdarstellung bietet dem (Fremd)Leser eine authentisch nahe - wenn auch fiktionale - Einsicht in die Fremdkultur. Die stark von der Globalisierung beeinflusste Welt, die unzählbaren digitalen Zugänge zu fremden Welten und Kulturen ermöglichen zwar eine kulturelle Überschreitung und Öffnung zur Fremde; gleichzeitig können gerade diese Chancen einperspektivisches Denken und falsche Bilder von der Fremde prägen. Diese Auffassung muss für die akademische Tätigkeit erhellt und für didaktische Konsequenzen curricular zusammengeführt werden. Die Vermittlung von Literatur in Form literarischer Werke und literaturwissenschaftlicher Inhalte gehört einmal mehr aus diesem Grund an ägyptischen Universitäten zu den Bausteinen der germanistischen Ausbildung. Sie ist eine Selbstverständlichkeit in der Germanistik überhaupt und eine essentielle curriculare akademische Vorgabe, die die Kenntnis von literaturwissenschaftlichen Inhalten beim Absolventen festsetzt. Mit

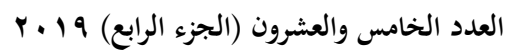

مجلة كلية التربية- جامعة عين شمس 
Literatur im Rahmen akademischer Vorgaben der Germanistik-

Ausbildung: Ästhetische Impulse und didaktische Outcomes

anderen Worten: Ein Germanistik-Studium ohne Literatur und Literaturwissenschaft ist undenkbar, unmöglich - kein GermanistikStudium.

Vor diesem Hintergrund setzt sich der vorliegende Beitrag zum Ziel, zunächst die Funktion und Rolle von Literaturvermittlung im Fremdsprachenunterricht innerhalb anerkannter Referenzrahmen zu erhellen. Diese sind einmal mehr darauf bedacht, nicht nur das Sprachniveau und die Wissenskompetenz zu systematisieren, sondern auch die Lernenden effizient für den Arbeitsmarkt vorzubereiten. Als dann werden aktuelle methodische Ansätze und ihre literaturdidaktischen Umsetzungen ins Visier genommen, die sich der Realisierung dieser beiden Zielsetzungen zur Aufgabe machen.

\section{Sinn der Literaturvermittlung in anerkannten ägyptischen und europäischen Referenzrahmen für den Fremdsprachenerwerb}

Ein ordnungsgemäßes, anerkanntes Studium an universitären ägyptischen Einrichtungen setzt vorgeschriebene Ziele, bei denen die Erkenntnisbereiche nicht allein durch Linguistik zu decken sind, sondern auch durch Literaturwissenschaft. Ein Referenzrahmen wie der der ägyptischen Qualitätssicherungsbehörde (NARS - National Academic Reference Standards) setzt hier klare Linien hinsichtlich der Kenntnisbereiche:

- Terminologie der Sprach- und Literaturwissenschaft

- Literarische Epochen und geistige Strömungen der Zielkultur

- Wichtige Dichter, Denker der Vergangenheit und der Moderne und ihre Werke

- Kulturelle, politische und gesellschaftliche Geschichte der Zielkultur

- Grundkenntnisse in Übersetzung und literarischen Theorien

- Humanistisches Wissen in Verbindung mit der Zielsprache. (vgl. NARS 2009: 3-6, Ü. d. V.) 
Ass.-Prof. Dr. Lamyaa Abdelmohsen Osman Ali Ziko

Diese Vorgaben gelten für eine universitäre, philologische Ausbildung in den unterschiedlichen Fremdsprachen. Die Studierenden und angehenden Absolventen werden ferner mit Grundkenntnissen in der arabischen Muttersprache bekannt gemacht, um diese auch für eine wechselseitige Kulturvermittlung nutzbar zu machen (vgl. ebd.).

Aber auch der GER (2001) erkennt im Zusammenhang mit Fremdsprachenerwerb die vermittelnde Funktion von Literatur an:

Nationale und regionale Literatur leistet einen wesentlichen Beitrag zum europäischen Kulturerbe; der Europarat betrachtet es als einen ,wertvollen gemeinsamen Schatz, den es zu schützen und zu entwickeln gilt". Literarische Studien dienen nicht nur rein ästhetischen, sondern vielen anderen erzieherischen Zwecken - intellektuell, moralisch und emotional, linguistisch und kulturell. (GER 2001: 62; H. d. V.)

Die Notwendigkeit von Literaturvermittlung liegt demzufolge nicht allein in der ästhetischen Wahrnehmung, sondern in den dadurch mit erzielten Absichten auf Ebene der Kognition, des Verhaltens, der Sprache und Kultur. Die Umsetzung dieser unterschiedlichen Kompetenzen sieht der GER in ,kreativen und ästhetischen Aktivitäten“. So gibt er unter Punkt „Ästhetische Sprachverwendung“" an:

Künstlerische und die Vorstellungskraft herausfordernde Verwendung von Sprache hat einerseits ihren eigenen Wert, andererseits ist sie auch von hohem Bildungswert. Kreative und ästhetische Aktivitäten können produktiv sein, rezeptiv, interaktiv oder sprachmittelnd (vgl. Abschnitt 4.4.4), und sie können sowohl mündlich als auch schriftlich sein, z. B.:

-Singen (Kinderreime, Volkslieder, Schlager usw.);

-Nacherzählen oder Nachschreiben von Geschichten;

-Anhören, Lesen, Schreiben und Sprechen fiktionaler Texte

(Geschichten, Reime usw.), einschließlich audiovisueller

Texte, Cartoons, Bildgeschichten usw.;

-Aufführung geschriebener oder improvisierter Stücke usw.;

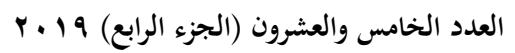

مجلة كلية التربية- جامعة عين شمس 


\section{Literatur im Rahmen akademischer Vorgaben der Germanistik-}

\section{Ausbildung: Ästhetische Impulse und didaktische Outcomes}

-Produktion, Rezeption und Aufführung literarischer Texte, $\mathrm{z}$. B.: Lesen und Schreiben von Texten (Kurzgeschichten, Romanen, Gedichten usw.), Aufführung und Rezeption von Liedern, Dramen, Opern usw. (ebd.: 61f)

Die Fähigkeit, eine Sprache ästhetisch zu verwenden, erfordert zunächst, ihre Ästhetik zu empfinden. Wie das geschieht oder wodurch, bleibt der Lehrkraft überlassen. Dem GER geht es vielmehr darum, dass die Fragen nach Lernen, Lehren und Beurteilen der ästhetischen (und spielerischen) Sprachverwendung im „Fragekasten“ erfüllt werden:

[...] was die Lernenden ,tun werden müssen oder wollen“, worauf sie ,vorbereitet werden sollen“ und welche „Anforderungen an sie gestellt werden“. (ebd.: 51)

Der Leitfaden der ägyptischen Qualitätssicherungsbehörde ersucht dies dagegen durch die Erfüllung von kognitiven, mentalen, praktischen und allgemeinen Kompetenzen (vgl. NARS 2009: 3-6).

Tatsächlich hat man sich bereits lange vor NARS oder GER Gedanken über den Sinn und über die Möglichkeiten der Literaturvermittlung im fremdsprachlichen Studium gemacht. NARS und GER fungieren in der gegenwärtigen und von der Globalisierung dominierenden Welt mehr als regulierende Richtlinien, die vor allem Vergleichbarkeit in der akademischen Ausbildung und gleichwertige Qualität garantieren wollen, wie auch eine Infrastruktur $\mathrm{zu}$ entwickeln beabsichtigen, die internationale Gültigkeit findet. Es haben sich schon früh zahlreiche theoretische Diskussionen und unzählige Umsetzungen von diversen theoretischen Ansätzen in der fremdsprachlichen Literaturdidaktik mit der Thematik Literatur im Fremdsprachenunterricht beschäftigt, woraus Lehrmaterialien für den fremdsprachlichen Literaturunterricht entwickelt wurden. Die Ausrichtungen hingen von den theoretisch-methodischen Grundlagen ab. Frühere zusammengestellte und erarbeitete Materialien setzten literarische Texte als Gegenstand für Spracharbeit oder zur Verfeinerung

العدد الخامس والعشرون (الجزء الرابع) 9 ا • ب

مجلة كلية التربية- جامعة عين شمس 
Ass.-Prof. Dr. Lamyaa Abdelmohsen Osman Ali Ziko

produktiver und rezeptiver Fertigkeiten ein und vernachlässigten dabei die ästhetische Wirkung des Textes. Die Bemühungen zielten später darauf ab, Literatur nicht als komplexes Sprachgebilde zu betrachten und aus dem Fremdsprachenunterricht $\mathrm{zu}$ verbannen, sondern als essentiellen und integralen Bestandteil des Vermittlungsprozesses von fremder Sprache und Kultur festzusetzen. Über die kommunikative Wende hinweg $\mathrm{zu}$ den rezeptionsästhetischen und dann interkulturellen Ansätzen sind literarische Texte nicht mehr nur als Gegenstand inhaltlichen Abfragens oder Aufnehmens von Informationen einzusetzen, sondern fordern den kreativen und deutungsoffenen Umgang mit Literatur (s. hierzu Helbig/Götze/Henrici/Krumm 2001; Wierlacher/Bogner 2003; Bausch/Christ/Krumm 2007). Mittlerweile versuchen Erzeugnisse moderner Ansätze, den Umgang mit Literatur im Fremdsprachenunterricht in Gleichgewicht zu halten: zwischen Ästhetik und Spracherwerb, Wissenschaft und Spracharbeit, Kulturspiegel und faktischem Wissen (s. bspw. Bischof/Kessling/Krechel 2003, Ehlers 2007; Dobstadt/Riedner 2017).

Bei näherer Betrachtung sind die didaktischen Überlegungen seit der Grammatikübersetzungsmethode über die kommunikative Wende und rezeptionsästhetische Überlegungen bis hin $\mathrm{zu}$ inter/transkulturellen Ansätzen mit ihren Kompetenzentwicklungen in der hiesigen Germanistik in den unterschiedlichen Studienjahren nutz- und einsetzbar. Sie ebnen den Weg zur Vermittlung von literaturwissenschaftlichem Wissen im fortgeschrittenen Studium, weil sie den unterschiedlichen Sprachniveaus der Studierenden angepasst werden können.

Ungeklärt ist allerdings, inwiefern ein fremdsprachlicher Literaturunterricht für den Germanistik-Absolventen von Nutzen sein könnte. NARS schreibt hier klare Zielsetzungen vor:

- Umsetzung des erworbenen Wissens im Beruf

- Richtiges Lesen zielsprachlicher Texte

- Übersetzung von Texten Arabisch/Fremdsprachlich

العدد الخامس والعشرون (الجزء الرابع) 9 ب ب r

مجلة كلية التربية- جامعة عين شمس 


\section{Literatur im Rahmen akademischer Vorgaben der Germanistik-}

Ausbildung: Ästhetische Impulse und didaktische Outcomes

- Richtiges Anwenden der Grammatik beim schriftlichen Verfassen von Texten oder Berichten, sowie bei der mündlichen Äußerung in der Zielsprache

- Führen von Gesprächen mit Muttersprachlern der Zielsprache. (vgl. NARS 2009: 5; Ü. d. V.)

Fremdsprachige Studiengänge sind jedoch nicht willkürlich, sondern zur Erfüllung bestimmter bildungspolitischer Bedürfnisse gegründet worden (vgl. ebd.: 1; GER 2001: 8-11). Die Beschreibungen dieser Fachrichtungen bzw. der Inhalte eines Fremdsprachenerwerbs in der Gegenwart - besonders nach dem Bologna-Abkommen - bauen im Grunde darauf auf, den neuen Erfordernissen entgegenzukommen. Die meisten Anforderungen versuchen, kommunikative Ziele auf der Ebene der rezeptiven und produktiven Fertigkeiten $\mathrm{zu}$ erreichen. Diese werden in den akademischen Einrichtungen curricular z. B. in Fächer wie Konversation, HV, Aufsatz oder ähnlichen Benennungen umgesetzt. Die Frage nach dem Sinn von Literatur und Literaturwissenschaft stellt sich in unserer Gegenwart umso mehr, als die Nachfrage nach Germanistik-Absolventen im (ägyptischen) Arbeitsmarkt gestiegen ist. Trotz dieser Vorgaben sind die Erfordernisse des Arbeitsmarkts in den Curricula nicht ausreichend ausgeschöpft, die zudem weit entfernt von einer literaturwissenschaftlichen Kompetenzausbildung sind.Worin besteht aber die Relevanz von Literatur und Literaturwissenschaft im Germanistikstudium für die angehenden Absolventen?

\section{Literaturwissenschaftliche Impulse und didaktische Outcomes}

Literatur ist ein ,sprachliches Kunstwerk“. Diese Bezeichnung Kaysers aus 1948 fasst die wesentlichen Merkmale eines literarischen Textes zusammen, wie sie auch in modernen Einführungen verstanden wird: künstlerische Sprachverwendung $(\approx$ sprachlich), Fiktionalität $(\approx$ Kunst) und Format bzw. Fixierung innerhalb eines Produktions- und Rezeptionsformat $(\approx$ Werk) (s.

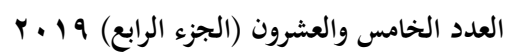

مجلة كلية التربية- جامعة عين شمس 
Ass.-Prof. Dr. Lamyaa Abdelmohsen Osman Ali Ziko

dazu auch Schneider 1998: 9-13, Culler 2002: 44-53 u.v.m). Das sind, abgesehen von den zu erwerbenden literaturwissenschaftlichen Erkenntnissen, gute Vorlagen für den fremdsprachlichen Literaturunterricht. Sie erfüllen Ziele des fremdsprachlichen Literaturunterrichts - Spracharbeit und ästhetische Erziehung - und beziehen kulturelle Inhalte ein.

Literatur als Erzeugnis zeitlicher und räumlicher Gegebenheiten birgt in sich zahlreiche Hintergründe: Literaturgeschichte, Entstehungsgeschichte, Biographie geben Einsicht in die ,Zielliteratur', die dem Nicht-Muttersprachler fremd ist. Sich Wissen darüber anzueignen, erfordert ein Verständnis dafür zu entwickeln. Dadurch könnten die Studierenden in der Lage sein, sich auf das Fremde einzulassen, seine Perspektive einzunehmen und evtl. auf die eigene Situation, im Sinne eigene Geschichte, eigene historische Ereignisse, $\mathrm{zu}$ projizieren. Auf inhaltlichanalytischer Ebene veranschaulicht die Behandlung und Lektüre von literarischen Werken hauptsächlich, dass Literatur eigentlich universell ist. Themen wie Liebe, Freiheit oder Rache können unterschiedlichen kulturräumlichen Stoffen oder Motiven entspringen, arbeiten manchmal aber auch nach demselben Muster. Wesentlich ist, dass die Studierenden Gemeinsamkeiten und Unterschiede wahrnehmen: das Menschliche, Private, Individuelle und das Kulturelle, Öffentliche, Kollektive. Was wem zuzuordnen ist, ist nicht festsetzbar, da es von Inhalt, Zeit, Ort, Produzenten und Rezipienten abhängt - und das macht Literatur gerade so interessant. Die Kenntnis über Gattungen erfordert ferner nicht nur charakteristische Merkmale von bestimmten Gattungen und Genren $\mathrm{zu}$ verstehen, sondern ist vorrangig Textsortenwissen. Literatur und Literaturwissenschaft sind in diesem Sinne nicht zum Selbstzweck eingeführt; sie erfüllen praxisbezogene Kompetenzen, die weitere Kompetenzen in einer späteren beruflichen Tätigkeit ebenen.

Ein erneuter Blick auf die NARS zeigt, dass die Absolventen im Allgemeinen während des philologischen Studiums folgende Fähigkeiten zu erwerben haben:

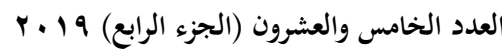

مجلة كلية التربية- جامعة عين شمس 


\section{Literatur im Rahmen akademischer Vorgaben der Germanistik-}

\section{Ausbildung: Ästhetische Impulse und didaktische Outcomes}

- Wissenschaftliches Denken und Arbeiten

- Kritisches und logisches Denken

- Nützliches Anwenden von Wissenschaft für sich und für die Gesellschaft

- Lernen aus dem Vergangenen für die Gegenwart und nachhaltiges zukunftsorientiertes Denken (vgl. NARS 2009: 8f; Ü. d. V.)

Die Relevanz eines Faches besteht hauptsächlich in der positiven Anteilnahme an Gesellschaft, ihrem Aufbau und ihrer Entwicklung. Letztendlich gehören $\mathrm{zu}$ jenen Erfordernissen und Zielen auch interkulturelle Kompetenzen wie bürgerliche Kompetenz und Fremdverstehen (vgl. ebd.: 9). Das sind Fertigkeiten, die nicht allein durch Spracharbeit und Landeskunde zu erwerben oder zu stärken sind.

Die germanistischen Lehrkräfte in Ägypten sind sich der oben genannten Tatsachen bewusst und haben für sich Strategien entwickelt, um das literaturwissenschaftliche Fach für die Absolventen sinnvoll $\mathrm{zu}$ gestalten und berufsrelevante Kompetenzen indirekt in den literaturwissenschaftlichen Unterricht einzubinden. In den Lehrveranstaltungen wird dabei insbesondere die sprachliche Darstellungsweise des literarischen Textes ermittelt, um auf die Ausdrucksstärke der vermittelten Inhalte, Intentionen, Bedeutungsvielfalt im literarischen Werk aufmerksam zu machen. Das ist für den Fremdsprachenlerner umso wichtiger, als er dadurch zwei Komponenten des Lernens zusammenführen kann: die wissenschaftliche und die fremdsprachliche. Ziel ist, durch den sprachlichen Ausdruck, die sprachlichen Einheiten eines literarischen Textes, sollten sie in der Lage sein, dessen Eigenart zu erkennen: Z. B. den Aufbau eines Gedichts, dessen Verse vermischt sind, durch das erkannte Reimschema wieder richtig zu ordnen; das Heben und Senken der Stimme, das langsame oder schnelle Lesen, was auch eine interpretierende Funktion haben könnte; oder die räumliche Figurenverteilung einer erfundenen und vorgespielten

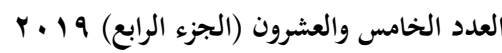

مجلة كلية التربية- جامعة عين شمس 
Ass.-Prof. Dr. Lamyaa Abdelmohsen Osman Ali Ziko

Szene, bei der auch die Relation der Figuren zu einander erkannt werden soll. Die angeführten Beispiele sind aus dem literaturwissenschaftlichen Unterricht, bei dem Spracharbeit und Fachwissen der Literaturwissenschaft integriert und in produktive Auseinandersetzung umgesetzt werden (dazu Ziko 2012 und 2013). Hauptanliegen ist, die Studierenden auf das Literarische, Ästhetische des Textes aufmerksam zu machen, ihre Hemmung in Bezug auf fremdsprachliche Literatur $\mathrm{zu}$ überwinden, die Trockenheit des theoretischen Wissens aufzufrischen und ihre Sprache innovativ zu gestalten lernen. In Ausnahmefällen hilft der Erwerb von literaturwissenschaftlichem Wissen Erzähltechniken in muttersprachlichen Werken zu verstehen. „Fachspezifische“ wie auch „überfachliche Ziele“ (Zeuner 2002: 530) sind demzufolge für eine akademische germanistische Ausbildung angestrebt. Herausforderungen wie die Stärkung der mündlichen Fertigkeit, die Regenerierung der studentischen Motivation, außerinstitutionelle Alternativen bzw. Fazilitäten können teilweise dadurch entgegengewirkt werden, indem ein Zusammenwirken verschiedener Veranstaltungen der unterschiedlichen Fachbereiche wie Linguistik und Übersetzung, aber auch fachübergreifende Lehrveranstaltungen erreicht wird (dazu Shalaby 2002/2003).

Galt es im vorherigen Teil des vorliegenden Beitrags Einblicke in die akademischen Voraussetzungen und Ziele eines GermanistikStudiums in Ägypten zu verschaffen und die Funktion von Literatur und Literaturwissenschaft innerhalb dieses Studiums einzuleiten, widmet sich der folgende Teil der Auseinandersetzung mit neuen didaktischen Überlegungen, die sich zur Aufgabe machen, die ästhetische Wirkung literarischer Texte im fremdsprachlichen Literaturunterricht hervorzuheben und/oder ein referenzgerechtes Konzept dafür $\mathrm{zu}$ erstellen, der den Erfordernissen des Spracherwerbs gerecht werden will.

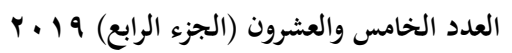

مجلة كلية التربية- جامعة عين شمس 


\section{Literatur im Rahmen akademischer Vorgaben der Germanistik-}

Ausbildung: Ästhetische Impulse und didaktische Outcomes

\section{Ästhetisierung und Didaktisierung des fremdsprachlichen Literaturunterrichts}

Der Bezug zwischen Spracharbeit und Literatur im Fremdsprachenunterricht mit Deutsch ist in den letzten Jahrzehnten immer mehr in das Zentrum der Diskussionen und didaktischen Umsetzungen gerückt. Literatur wird in diesen nicht mehr als Redeanlass, Abfrage von Inhalten oder sonstigem betrachtet; das Augenmerk der Lernenden wird auf das Ästhetische, Literarische gelenkt; theoretische, konzeptionelle Gedanken werden mit der fremdsprachlichen Literaturvermittlung verbunden (vgl. hierzu Weinrich 1983; Honnef-Becker 1993; Bredella 2002; Ehlers 1989 und 2007; Mecklenburg 2003; Tütken 2006; Rösch 2011; Altmayer/Dobstadt/Riedner 2014; Dobstadt/Riedner 2017, u.v.m.). Literatur soll wieder Spaß machen, (sprach)spielerisch angegangen werden und nicht hemmend, abschreckend wirken (vgl. bspw. Weinrich 1983 - „mit Phantasie“; Honnef-Becker 1993 - „mit Vergnügen“). Zu diesen didaktischen Bemühungen zählt neben der erstmals 1992 erschienenen Fernstudieneinheit von Swantje Ehlers Lesen als Verstehen (2007) unter anderem das hier zu untersuchende Materialband Literatur Lesen Lernen (2017) der Herausgeber Dobstadt/Riedner.

Ehlers bemüht sich in der Fernstudieneinheit um das bereits im Titel propagierte Lesen als Verstehen, das vor allem mit literarischen Texten gelernt werden soll. Verstehen erweist sich als ein annähernder Prozess, durch den nicht nur der literarische Text erschlossen wird, sondern stufenweise auch Pointe, Intention, charakteristische Merkmale der Figuren und Perspektiven, die der Text aufweist, erarbeitet werden (vgl. Ehlers 2007: 6ff.). Zudem könnten „Verallgemeinerungen des Textes und Einordnungen in den entsprechenden gesellschaftlichen Zusammenhang“ ${ }^{\text {zu }}$ „Vergleichen mit und/oder Abgrenzen von der Gesellschaft des jeweiligen fremdsprachigen Lesers" (ebd.: 8) führen. Diverse Zugänge zum Verstehensprozess eines literarischen Werkes werden

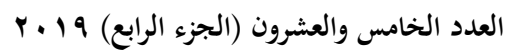

مجلة كلية التربية- جامعة عين شمس 
Ass.-Prof. Dr. Lamyaa Abdelmohsen Osman Ali Ziko

in dieser Fernstudieneinheit geöffnet, die zum einen die Rolle der Lehrkraft aufzeigt, zum anderen aber die Möglichkeiten der aktiven Beteiligung des Lerners als Leser am Verstehensprozess zur Schau stellt. Ehlers hebt in diesem Zusammenhang auch die Relevanz der ästhetischen Wahrnehmung von Formen und Darstellungsweisen des literarischen Textes als unumstrittener Bestandteil des Verstehensprozesses hervor. Die erworbenen „Deutungstechniken“ schulen interpretatorische Kompetenzen wie die Auswahl und das Bewerten von Faktoren innerhalb des Textes und deren Einbeziehen in den Gesamtkontext. Hinzu kommt der Perspektivenwechsel, wodurch die Lerner ,neue Aspekte in Betracht ziehen und zu neuen Einordnungen gelangen" (ebd.: 49), die sich mitunter auf die eigene Erfahrungswelt beziehen könnten („Applikation des Textes“, vgl. ebd.: 48).

Das Materialband Literatur Lesen Lernen von Dobstadt/Riedner macht diesen Aspekt der ästhetischen Wahrnehmung zum Schwerpunkt. Es ist für das Sprachniveau B2 gedacht und will vorrangig den Deutschlernenden auf die Literarizität des literarischen Textes aufmerksam machen und ihn dadurch anregen, sich reflexiv mit Sprache auseinanderzusetzen (vgl. Dobstadt/Riedner 2017: 8ff). Der Ansatz beruht dabei hauptsächlich auf der „symbolische[n] Kompetenz“ von Kramsch (2011).

In ihrem Beitrag Symbolische Kompetenz durch literarische Texte schlägt Kramsch vor, beim Umgang mit literarischen Texten im DaF-kommunikativ-interkulturell ausgerichteten Ansatz durch eine symbolische Kompetenz zu erweitern. Hier soll sich der Lernende über die Machart, die Ausdrucksweise des literarischen Textes bewusst werden, also erkennen, wie sich ,,poetische, gefühlsmäßige und ideologische Dimensionen" sprachlich gestalten und auf den Verstehensprozess, auf die Deutung und Interpretation auswirken. Literarische Texte fungieren in diesem Sinne nicht mehr nur als eine Möglichkeit, einen Sprechanlass auszulösen, worin Meinungen geäußert, Informationen ausgetauscht oder Stellung zu bestimmten Themen genommen werden. Dieser Vorgang soll den

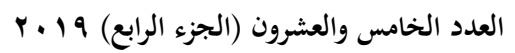

مجلة كلية التربية- جامعة عين شمس 


\section{Literatur im Rahmen akademischer Vorgaben der Germanistik-}

\section{Ausbildung: Ästhetische Impulse und didaktische Outcomes}

Fremdsprachenlerner dazu befähigen, festbestehende Meinungen zu lockern und nicht nur aus der eigenen Überzeugung sich zu einem Text zu äußern (vgl. Kramsch 2011: 36). Ziel eines derartigen ästhetisch-orientierten Verfahrens ist, die Literarizität des literarischen Textes - oder wie Schiedermair (2011) ihren Beitrag tituliert dadurch, „[1]iterarische Texte als literarische Texte“ zu erkennen. Der Lerner soll für die Wahrnehmung der sprachlichen Ausdruckskraft des literarischen Textes sensibilisiert werden, mit dem weiteren Ziel, bei ihm einen Perspektivenwechsel zu bewirken, der die Sichtweise des Anderen, Fremden einzunehmen und einzufühlen hilft.

Kramsch nennt drei Aspekte der symbolischen Komponente, die sich mit den drei Funktionen von Sprache bei Bühler identifizieren lassen: Darstellung, Appell und Ausdruck (vgl. Kramsch 2011: 36). Hierin werden auch die drei wesentlichen Merkmale des literarischen Textes realisiert, nämlich: Anschaulichkeit, Vieldeutigkeit und Kontextverbundenheit (s. Schiedermair 2011).

Die symbolische Darstellung, d.h. das, was Sprache aussagt, realisiert sich durch die denotative und konnotative Bedeutung von Wörtern, also durch ihre wörtliche Bedeutung und durch jene Bedeutung, die sich aus dem Kontext und aus der Perspektive des fremdsprachigen Lesers ergibt. Die symbolische Sprachhandlung oder auch Appell, also was die Sprache realisiert, nutzt bestimmte sprachliche Mittel, um den Rezipienten - in diesem Fall auch der Fremdsprachenlerner - auf sich aufmerksam zu machen. Sie deutet bspw. auf die Textsorte bzw. auf das literarische Genre hin. Dabei lenkt man auf typische Merkmale eines bestimmten Genres, z. B. kurze Sätze und Wiederholungen in Kurzgeschichten etc. Der symbolische Ausdruck, womit gemeint ist, was durch Sprache evoziert wird, bezieht sich auf die Wirkung der angewandten sprachlich-literarischen Mittel wie Klang, Metapher, um Resonanzen und Assoziationen auszulösen. Kramsch räumt hier allerdings auch ein, dass diese Fokussierung auf die Machart eines

العدد الخامس والعشرون (الجزء الرابع) 9 ا ـ r

مجلة كلية التربية- جامعة عين شمس 
Ass.-Prof. Dr. Lamyaa Abdelmohsen Osman Ali Ziko

literarischen Textes vor allem das Verdienst der Rezeptionsästhetik sei. Der Vorteil der Wahrnehmung von Vieldeutigkeit, Anschaulichkeit und Kontextverbundenheit der literarischen Texte sei die Entlarvung fester Kategorien, die sich $\mathrm{zu}$ dynamischen entwickeln können. Das ermögliche ein prozesshaftes flexibles Reagieren auf eine sich verändernde Welt. Die Lerner lassen und stellen sich auf diese Bandbreite polysemer, kontextueller Möglichkeiten ein (vgl. Kramsch 2011: 36ff.). Diesen Ansatz greifen die Herausgeber und Verfasser des Materialbandes Literatur Lesen Lernen auf und entwickeln dafür didaktische Modelle für den Umgang mit Literatur.

\section{Das didaktische Konzept des Materialbandes Literatur Lesen} Lernen

Das Materialband präsentiert sich auf dem Deckblatt als „Lesewerkstatt“", einsetzbar im Erst-, Zweit- und Fremdsprachenunterricht und konfrontiert in der Einleitung mit der bewussten Abweichung von der traditionellen Trennung und Ausdifferenzierung zwischen Deutsch als Mutter-, Zweit- und Fremdsprache im Hinblick auf die Behandlung von Literatur im Deutschunterricht. Der Grund dafür sei das sich durch Globalisierung verändernde Weltbild, aber auch die Vielsprachigkeit und die ,medialen und wirtschaftlichen Verflechtungen“ (Dobstadt/Riedner 2017: 6) in der Weltgemeinschaft. Das wiederum habe seinen Einfluss auf die Wahrnehmung von Fremd- und Eigenkultur (vgl. ebd.). Hier wird die Transkulturalität aufgegriffen, die das Weltbild prägt und worin sich hybride Gebilde, Charaktere, Gesellschaften entwickelt haben, die in sich unterschiedliche Kulturen tragen und diese miteinander harmonisch bringen (können). Diese Entwicklungen, so Verfasser und Herausgeber, stellen in diesem Sinne die „Entgegensetzung von Fremd- und Eigenkultur" in Frage, was wiederum auf die Perspektive einwirkt, wie Mutter- und Fremdsprachenunterricht zu realisieren sei. Nicht zuletzt sei die Sprache von Literatur für den

العدد الخامس والعشرون (الجزء الرابع) 9 ا ـ r

مجلة كلية التربية- جامعة عين شمس 


\section{Literatur im Rahmen akademischer Vorgaben der Germanistik-}

Ausbildung: Ästhetische Impulse und didaktische Outcomes

Muttersprachler „immer auch ein Stück Fremdsprache“, die dem Muttersprachler nicht immer erschließbar sei (vgl. ebd.).

Diese ,neue، literaturdidaktische Betrachtung von Literatur im Mutter- wie Fremdsprachenunterricht lenkt das Augenmerk auf ein wesentliches Merkmal literarischer Texte, nämlich auf seine künstlerische Sprachverwendung - eine Tatsache, die in zahlreichen älteren und modernen literaturwissenschaftlichen Einführungen immer wieder bestätigt wird (Kayser 1968; Schneider 1998; Culler 2004 u.a.), die jedem Literaturwissenschaftler im ,literarischen Fremdsprachenunterricht bewusst ist und die er seinen Lernern bewusst $\mathrm{zu}$ machen versucht. Das gilt vor allem auch für den Fremdsprachler, der wahrnehmen sollte, dass das Nicht-Verstehen eines literarischen Textes nicht allein auf die fremde Sprache zurückzuführen ist, sondern vorrangig auf seine Wesensart, seine polyseme Dimension, seine Ästhetik und seine Literarizität.

Letzterer Begriff ist das Hauptanliegen der Erstellung dieser ,Lesewerkstatt', mit dem die Verfasser und Herausgeber auch dem GER entgegen zu kommen beabsichtigen, dessen Vorgaben, „Testbarkeit, Messbarkeit, Effizienzorientierung“ und „Verwertbarkeit“, einen distanzierten Umgang mit literarischen Texten im Fremdsprachenunterricht bewirkten (vgl. ebd.: 7). Vor dem Hintergrund der PISA-Tests erkannte man vor allem im muttersprachlichen Literaturunterricht den Vorteil der Literatur. Sie wurde ,als Chance für die Bekämpfung der in den PISA-Tests zutage getretenen Schwäche der Schülerinnen und Schüler beim Lesen und Verstehen komplexerer Texte gesehen" (ebd.).

Das Konzept der literarischen Spracharbeit in diesem Materialband baut neben der Rezeptionsästhetik auf die in den USA und Europa entwickelte „Second Language Aquisistion (SLA)-Forschung“, die eine Spracharbeit anstrebt, die auf ,einer literarischen und auf den Erwerb symbolicher Komptenz" gegründet ist (ebd.). Es lenkt die Aufmerksamkeit auf die strukturelle Ebene des literarischen Textes, wodurch sich das Blickfeld nicht nur auf die vielseitige

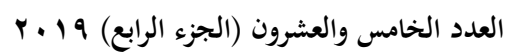

مجلة كلية التربية- جامعة عين شمس 
Ass.-Prof. Dr. Lamyaa Abdelmohsen Osman Ali Ziko

Deutungsmöglichkeit des Inhalts und Ausgesagten erweitert, sondern auch auf die sprachliche Komposition des Inhalts und Ausgesagten - also Form und Verfasstheit des Textes (vgl. ebd.: 8). Das didaktische Modell in den unterschiedlichen Einheiten des Matieralbandes Literatur Lesen Lernen verfolgt das Ziel, den Lerner für Literatur $\mathrm{zu}$ sensibilisieren und will ferner durch Literatur Fähigkeiten vermitteln, die im Umgang mit nicht-literarischen Texten hilfreich sind. Die Vorteile literarischer Texte sehen die Herausgeber darin, dass sie einen vertieften und reflektierten Umgang mit Literatur erfordern und einen kreativen und produktiven Umgang mit Sprache erlauben. Ferner wird der Lernende ermutigt, einen eigenen sprachlichen Ausdruck zu entwickeln. Dazu sind Techniken zu erwerben, wie Modifikation, Veränderung, Zusammensetzung usw. Diesbezüglich betonen die Herausgeber, dass die Kenntnis über literarische Ausdrucksformen und Elemente dabei dienlich seien: Gattung, Motiv, Plot, Erzählund Darstellungsweise, aber auch die sprachliche Textverbindung. Damit bietet Literatur ein Potential für Training und Übung, gleichzeitig ist ein „Mehrwert für den Deutschunterricht“ daraus zu erschließen. Die literarische Sprache trete nicht als System auf. Ihre Normverstöße und Abweichungen würden Lust an Sprache erwecken und zum kreativen Umgang anregen. Das wiederum sei eine Voraussetzung für die Entwicklung der eigenen Ausdrucksfähigkeit. Damit beschränkt sich die Kommunikation nicht nur auf Bedeutungsvermittlung, sondern trägt in sich auch bedeutungsbeeinträchtigende Sinnlichkeit, woraus sich die Deutungskompetenz des Fremdsprachenlerners entfalten kann und gleichzeitig eigene Schemata und Muster wiedererkennen lassen. Schließlich realisiert der Fremdsprachenlerner, dass Literatur nicht allein für sich stehe, sondern auch innerhalb ,diskursive[r] Zusammenhänge[...]“" (vgl. ebd.: 9f).

Vor diesem Hintergrund machen die Herausgeber des Materialbandes darauf aufmerksam, ein weitgefasstes Verständnis von Literatur zu haben, denn das Materialband zieht neben den

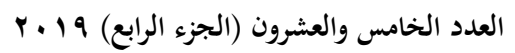

مجلة كلية التربية- جامعة عين شمس 


\section{Literatur im Rahmen akademischer Vorgaben der Germanistik-}

Ausbildung: Ästhetische Impulse und didaktische Outcomes

üblichen literarischen Texten auch Filme bzw. Filmausschnitte, Drehbuch und Comics heran (vgl. ebd.: 10). Es werden zudem Textsorten wie Plakat, Werbetext, Ankündigungen und Filmbesprechungen, Leserbriefe und Rezensionen eingebunden, die für die sprachliche und kreative Auseinandersetzung in den thematischen Schwerpunkten eingesetzt werden.

\section{Integration der symbolischen Kompetenz im Aufbau der Unterrichtseinheiten}

In der Einführung des Materialbandes wird die Gliederung der Arbeit mit den ausgewählten literarischen Texten in den zehn von einander unabhängigen Einheiten umrissen. Die Umsetzung der symbolischen Kompetenz erfolgt integrativ in unterschiedlichen rezeptiv und produktiv orientierten Arbeitsformen in drei aufeinander bauenden Phasen. Die erste Phase ,gilt der ersten Begegnung mit dem Text" (ebd.: 11). Sie setzt sich unmittelbar mit dem Text auseinander und will zunächst aus dem Text heraus dem Lerner das Verstehen des Textes näher bringen. Es wird in den meisten Einheiten auf eine Vorentlastung verzichtet, was dem Wesen der Literatur entgegenkommt: Beim Lesen von literarischen Texten lässt man sich ohnehin bewusst auf das Unerwartete ein. „Irritationsmomente in literarischen Texten" sind also erwünscht und wollen nicht vorweg angekündigt werden. In dieser Phase wird der Lerner a) auf ,die Form und die bedeutungsbildende Kraft“ des literarischen Textes aufmerksam, b) dazu aufgefordert, „Leseeindrücke zu sammeln“ und c) erlebt er meistens durch das Lesen von Textauszügen eine „sinnliche Erfahrung“ des Textes. Hauptsächlich ist es wichtig, dass der Lerner d) nicht von der Lehrkraft gesteuert oder beeinflusst wird (vgl. ebd.: 12). Die Bewusstwerdung, dass sich die Erfahrung des literarischen Textes sozusagen ,intim' oder wenigstens, individuell' vollziehen kann, ist in diesem Fall von besonderer Relevanz. Die Lesewerkstatt bedient sich in dieser Phase derartigen Aufgaben, die dem Lerner die ersten

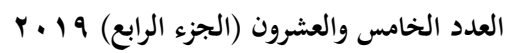

مجلة كلية التربية- جامعة عين شمس 
Ass.-Prof. Dr. Lamyaa Abdelmohsen Osman Ali Ziko

Emotionen, Eindrücke und/oder Erwartungen entlocken sollen. Sie setzen sich zudem mit der Wirkung der Sprache auseinander: die Relation zwischen den Wörtern, der Sinn von Satzzeichen, Assoziierungen in Form von Assoziogrammen oder Standbildern, Tabellen und Kategorien erstellen, Eindrücke zu Klang und Rhythmus durch lautes (Vor)Lesen, Besonderheiten sprachlicher Ausdrücke etc.

Die zweite Phase arbeitet „an und mit dem Text“" (ebd.: 11). Die Auseinandersetzung mit dem Text geschieht durch Aufgaben, die das Sprachbewusstsein vertiefen, fördern und die Deutungsmöglichkeiten klar machen. Lerner sollen „Lust am Lesen" (ebd.: 12) und am Proben mit der Sprache entwickeln. Ein Weg dazu ist die Produktion von Texten, wodurch die Lerner die Texte erneut rezipieren. Das wird erreicht durch zwei Arbeitsformen: Transformationsaufgaben und Text im Kontext. Bei der Transformationsaufgabe handelt es sich um das Umsetzen in eine andere nichtliterarische Textsorte, wie Zeitungsartikel, Dialoge, Rezension u.ä.; oder das Einnehmen eines Perspektivenwechsels, von dem aus sich auch die Wahl eines angemessenen Ausdrucks und eines passenden sprachlichen Mittels üben lässt. Viele der Transformationsübungen sind aber vornehmlich darauf bedacht, das kreative Schreiben zu fördern. Durch die Vermittlung literarischer Techniken des Schreibens werden die Lerner dazu ermutigt, eigene kurze Geschichten oder Gedichte zu verfassen. In Text im Kontext werden die literarischen Texte im Zusammenhang der gesellschaftlichen Diskurse betrachtet, in denen sie entstanden sind. Es werden vorwiegend kurze Biographien der Autoren eingesetzt, worin auch bestimmte thematische Probleme angesprochen werden, die der Ausweitung der produktiven Auseinandersetzung mit dem behandelten Schwerpunkt dienen. Mit diesen Aufgabenformen erzielt das Materialband zum einen, dass das Bewusstsein für den Einfluss von Kontextwissen auf die eigene Deutung geschärft wird; zum anderen eröffnet dieses quasi hermeneutische Verfahren den Lernern neue Zugänge, den Text erneut und tiefer zu verstehen. Das

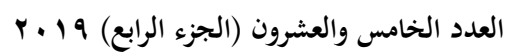

مجلة كلية التربية- جامعة عين شمس 


\section{Literatur im Rahmen akademischer Vorgaben der Germanistik-}

Ausbildung: Ästhetische Impulse und didaktische Outcomes

wiederum provoziert zu neuen Deutungen, die weitere sprachliche Äußerungen evozieren.

In der dritten und letzten Phase werden „Ergänzungs- und Erweiterungstexte eingeführt" (ebd.: 11). Es kommt dabei zu Textvergleichen mit thematisch ähnlichen Texten, mit dem Ziel, den Lernenden für unterschiedliche Ausdrucksmöglichkeiten zu sensibilisieren, die die deutsche Sprache in ihrer Vielfalt und ihren Facettenreichtum zeigt.

Die drei Phasen versuchen demzufolge den Forderungen Anschaulichkeit, Kontextverbundenheit und Vieldeutigkeit nachzukommen: durch „Modifikation, Rekombination, Veränderung und Neuzusammensetzung“ sollen „sprachliche Innovationen als Voraussetzung einer solchen Entwicklung“" (ebd.: 9), also des eigenen Ausdrucks, erzeugt werden. Die Verbesserung und Festigung der sprachlichen Produktion - Spracharbeit - ist das angekündigte Ziel des Materialbandes und erfolgt durch die Verlagerung der Arbeit mit literarischen Texten von der Abfrage nach Inhalten auf die Wahrnehmung des Ästhetischen, des Symbolischen, des Literarischen.

\section{Literatur Lesen Lernen: Ästhetische Lösungen für literaturdidaktische Fragen?}

Nach dieser Darstellung des didaktischen Konzepts des Materialbandes Literatur Lesen Lernen von Dobstadt/Riedner stellt sich die Frage, welchen Stellenwert es innerhalb der literaturdidaktischen Bemühungen besitzt und inwieweit es die erwünschten Anforderungen der besprochenen Referenzrahmen erfüllt.

Das Materialband bietet zweifelsfrei durchdachte Leitlinien für die Didaktisierung der gewählten literarischen Texte und realisiert dadurch mehrere Ziele:

- Es erleichtert - wie es selbst die Heraugeber beabsichtigen solchen Lehrkräften, die nicht literaturwissenschaftlich

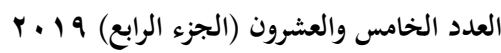

مجلة كلية التربية- جامعة عين شمس 
Ass.-Prof. Dr. Lamyaa Abdelmohsen Osman Ali Ziko

ausgebildet sind, den literaturdidaktischen Umgang in einem fremdsprachlichen Sprachunterricht. Das allein ist ein Indiz dafür, dass die Lesewerkstatt vielmehr für solche Seminare einsetzbar ist, die sprachliche Fertigkeiten üben und vertiefen wollen.

- Die Konzentration auf die symbolische Kompetenz ausgerichtete Spracharbeit mit literarischen Texten soll eine Rückbesinnung seiner Literarizität auslösen und damit auf seine wesentliche Eigenart (die künstliche Sprachverwendung) aufmerksam machen.

- Das Materialband hebt in diesem Zusammenhang vor allem das Sprachspielerische in den literarischen Texten hervor, das sprach- und kulturreflexives Potential in sich birgt. Dieses „Potential“, so die Meinung der Herausgeber, „eines solchen literarischen Umgangs mit Text und Sprache für fremdsprachliches - sprachliches wie kulturbezogenes Lernen blieb unausgeschöpft“" (ebd.: 8). Die Lesewerkstatt strebt in diesem Sinne den Versuch an, eine Lücke im fremdsprachlichen Literaturunterricht $\mathrm{zu}$ schließen und gleichzeitig die Auffassung von Referenzrahmen wie GER über Sprachvermittlung und Literatur umzusetzen.

- Diese Ausrichtung sensibilisiert den Lerner nicht nur dazu, Literatur $\mathrm{zu}$ rezipieren, sondern motiviert ihn $\mathrm{zu}$ einem kreativen Umgang mit Sprache.

Literaturrezeption und sprachliche Kreativität werden in den zehn Einheiten dieser Lesewerkstatt mittels unterschiedlicher Aufgaben während des Verstehensprozesses und der thematischen und sprachlichen Auseinandersetzung mit dem Text vollzogen:

- Die Verfasstheit des Textes, seine Literarizität, erfolgt durch eine Begegnung mit dem Text, die auf der Wahrnehmung und Versprachlichung sinnlicher und emotionaler Eindrücke beruht.

- Diese werden weiter mittels Transformationsaufgaben, Text im Kontext und anderen Aufgabenformen, die die

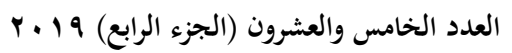

مجلة كلية التربية- جامعة عين شمس 


\section{Literatur im Rahmen akademischer Vorgaben der Germanistik-}

Ausbildung: Ästhetische Impulse und didaktische Outcomes

Spracharbeit intensivieren sollen, für die kreative Produktion von Texten umgewandelt.

- Ferner fördern diese Aufgabenformen jene sprachlichen Kompetenzen, die für die spätere Anwendung von Sprache im Beruf notwendig sind: die Entwicklung eines (eigenen) sprachlichen Ausdrucks und Textsortenbewusstseins.

Erwünschenswerte Inhalte und Kompetenzen, die von den erwähnten ägyptischen und europäischen Referenzrahmen gefordert werden, sind hier wieder zu erkennen:

- Es wird literaturwissenschaftliches Basisgrundwissen über ausgewählte Dichter/Schriftsteller, Epochen oder literarische Gattungen eingeführt.

- Es werden aktuelle Themen deutschsprachiger Literatur hinsichtlich der zwischenmenschlichen Beziehungen behandelt.

- Diese wiederum reflektieren Kultur und Verhaltensformen der Zielsprache und Zielkultur und erfüllen letztlich dadurch erzieherische und intellektuelle Ziele.

- Schließlich werden mittels diverser Lernarrangements soziale Kompetenzen wie Teamfähigkeit durch Partner- und Gruppenarbeit, Zeitmanagement und auch Planen und Organisieren praktiziert.

In dieser Hinsicht kann festgestellt werden, dass die Lesewerkstatt von Dobstadt/Riedner eine lobenswerte Leistung nicht allein durch die didaktische Modellierung ist, sondern auch durch den Textreichtum und didaktischen Aufwand wie auch im Bestreben um Aktualität, d.h. in Bezug auf aktuelle Themen der deutschsprachigen Zielkultur und schließlich die Wiederführung in die Spracharbeit. Die symbolische Kompetenz als methodisch-didaktisches Fundament dient somit der Wahrnehmung des Literarischen, aber vor allem der Spracharbeit.

Die Verlagerung des Umgangs mit literarischen Texten auf die ästhetische Wahrnehmung des sprachlichen Ausdrucks ist

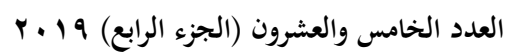

مجلة كلية التربية- جامعة عين شمس 
Ass.-Prof. Dr. Lamyaa Abdelmohsen Osman Ali Ziko

insbesondere im universitären, fremdsprachigen Literaturunterricht ein Bestandteil des literaturwissenschaftlichen Vermittlungsprozesses. Rezeptionsästhetische Ansätze, wie sie bspw. Ehlers in der Fernstudieneinheit einsetzt, unterscheiden sich im Vergleich mit dem untersuchten Materialband gering: von der Auseinandersetzung mit der Bedeutungsbildung der Wörter, über die Erstellung von Assoziogrammen, Mind Maps, Schreibprotokollen, bis hin zur Transformation in andere (literarische und nicht-literarische) Textsorten.

Die Ambitionen, durch das Materialband auch literaturwissenschaftliche Begriffe einzuführen, sind in jener Hinsicht problematisch, da diese hier nicht weiter erklärt werden ein weiteres Indiz dafür, dass als Begleitmaterial postuliert werden könnte. Die eingeführten Begriffe wie Erzählperspektive, Motiv usw. sind dem Lernenden hilfreicher, wenn er vorher erworbenes Fachwissen mitbringt und dadurch in den unterschiedlichen Einheiten sein sprachliches Vermögen vertiefen lernt. Mögliche Barrieren liegen nicht unbedingt im sprachlichen Ausdruck oder im sprachkünstlerischen Gebrauch literarischer Texte. StudentInnen, die eingeführt sind in das Wesen von Literatur, werden die Arbeitsmethoden nachvollziehen können.

\section{Fazit}

Die Wahrnehmung des ästhetischen und symbolischen Charakters eines literarischen Textes erfolgt im besprochenen Materialband Literatur Lesen Lernen von Dobstadt/Riedner, aber auch in der Fernstudieneinheit von Ehlers Lesen als Verstehen durch die Komponente des Lesens, die eine intensive sprachliche Auseinandersetzung zunächst rezeptiv und anschließend produktiv mit dem Text erlaubt. Diese Wahrnehmung kann ferner durch eine kognitive Auseinandersetzung mit literaturwissenschaftlichen Inhalten geschärft werden. Hier werden den Lernern - ganz im Wortlaut der Herausgeber des Materialbandes - „Texte in ihrer

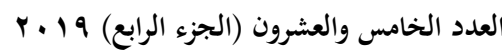

مجلة كلية التربية- جامعة عين شمس 


\section{Literatur im Rahmen akademischer Vorgaben der Germanistik-}

Ausbildung: Ästhetische Impulse und didaktische Outcomes

Komplexität und Vieldeutigkeit, mit anderen Worten: in ihrer Literarizität zugänglich" (ebd.: 8).

Dieser Zugang ist im fremdsprachlichen Literaturunterricht nicht ungewohnt, zumal die Fremdsprachenlerner bereits in den ersten Stunden des Fremdsprachenerwerbs mit der Vieldeutigkeit und Anschaulichkeit der Sprache konfrontiert werden und nehmen im fortgeschrittenen Studium die vielfältigen Möglichkeiten des sprachlichen Ausdrucks bewusst wahr. Dieser Ansatz der ästhetischen, symbolischen Auseinandersetzung mit Literatur und Sprache ist für den fremdsprachlichen Literaturunterricht an ägyptischen Universitäten gang und gäbe - auch wenn bis in die Gegenwart hinein eine theoretisch-didaktische Fundierung nicht ausreichend angegangen wurde (vgl. Ziko 2019: 77, 82). Es ist daher auch nicht verwunderlich, dass sich dieser Ansatz der symbolischen Kompetenz in den USA entwickelt hat, wo kulturwissenschaftliche Theorien verarbeitet und dabei die gesellschaftlichen und kulturellen Entwicklungen berücksichtigt wurden (dazu Kramsch 2016).

Tatsächlich ist jede Germanistik als DaF damit konfrontiert, im Gegensatz zur traditionellen Germanistik, mit Literatur anders umzugehen und innovative Ideen für den Einsatz von literarischen Texten und zur Vermittlung von Literaturwissenschaft im Rahmen der Vermittlung von Deutsch als Fremdsprache innerhalb einer universitären Einrichtung zu entwickeln. Die Abspaltung von DaF und Germanistik innerhalb des deutschsprachigen Raums ist ein langer Prozess gewesen; Diskussionen hierzu und zur Entwicklung der Schwerpunkte von DaF sind ebenso zahlreich als das sie hier auch nur angesprochen werden könnten. Es muss in diesem Zusammenhang darauf hingewiesen werden, dass die ägyptische Germanistik sich diesen theoretischen Diskussionen noch rezeptiv verhält und eigens keine theoretischen Konzepte vorschlägt, obwohl in der Praxis tatsächlich ähnliche Verfahren, wie sie hier vorgestellt

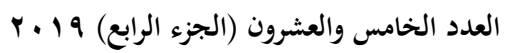

مجلة كلية التربية- جامعة عين شمس 
Ass.-Prof. Dr. Lamyaa Abdelmohsen Osman Ali Ziko

wurden, befolgt werden. Diese Lücke ist in der ägyptischen Germanistik zu füllen.

Die Notwendigkeit einer theoretischen Fundierung liegt ferner darin, Referenzrahmen, wie dem der ägyptischen NARS oder dem GER, gerecht $\mathrm{zu}$ werden, um akademische Ziele effektiv verwirklichen zu können und international eingebunden zu werden. Das universitäre Erlernen einer Fremdsprache ist vordergründig ein philologischer Wissenserwerb, der kognitionsbedingt ist. Sprache oder Literatur, als elementare Gegenstände des GermanistikStudiums in Ägypten, ist nicht zuletzt durch ein wissenschaftliches Inventar zu vermitteln und zu verstehen, das nicht nur sprach- oder literaturwissenschaftlicher Art ist, sondern auch kulturelles und historisches Wissen bedeutet.

In Anbetracht dessen bietet das Band Literatur Lesen Lernen m. E. zwar einen der vielen Wege, Sprache, Kultur, Wissen und berufsbezogene Kompetenzen - wie es die genannten Referenzrahmen vorsehen - zusammenzuführen. Allerdings muss hier auch eingeräumt werden, dass es keine Alternative für den literaturwissenschaftlichen Vermittlungsprozess darstellt, der institutionell und akademisch erforderlich ist, jedoch einsetzbar in weiteren curricularen Angeboten, die vor allem kommunikative und soziale Kompetenzen erreichen wollen.

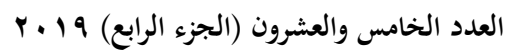




\section{Literatur im Rahmen akademischer Vorgaben der Germanistik-}

\section{Ausbildung: Ästhetische Impulse und didaktische Outcomes}

\section{Literatur}

Ali, Adel 2009: Bildergeschichten im DaF-Unterricht. Förderung sprachlicher Kompetenz und Kreativität. In: Info DaF 36/5, S. 462478.

Altmayer, Claus 2008: Was ist deutsche Kultur? Zum Gegenstand der Kulturstudien. In: Martinson, Steven D./Schulz, Renate A. (Hrsg.): Deutsch als Fremdsprache. Brücken bauen. Jahrbuch für internationale Germanistik. Bd. 94. Bern; Berlin; Bruxelles; Frankfurt am Main; New York; Oxford; Wien: Peter Lang. S. 2537.

Altmayer, Claus/Dobstadt, Michael/Riedner, Renate 2014: Literatur in sprach- und kulturbezogenen Lehr- und Lernprozessen im Kontext von $\mathrm{DaF} / \mathrm{DaZ}$. Eine Einführung in den Themenschwerpunkt. In: Deutsch als Fremdsprache, 51/1, S. 3-10.

Bausch, Karl-Richard/Christ, Herbert/Krumm, Hans-Jürgen 2007 (Hrsg.): Handbuch Fremdsprachenunterricht. 5. Aufl. Tübingen; Basel: A. Franke Verlag.

Bischof, Monika/Kessling, Viola/Krechel, Rüdiger 2003: Landeskunde und Literaturdidaktik. Fernstudieneinheit 3. Berlin; München; Wien; Zürich; New York: Langenscheidt.

Bredella, Lothar 2002: Literarisches und interkulturelles Verstehen. Tübingen: Gunter Narr Verlag.

Casper-Hehne, Hiltraud/Middecke, Annegret 2009 (Hrsg.): Sprachpraxis der DaF- und Germanistikstudiengänge im europäischen Hochschulraum. Göttingen: Universitätsdrucke Göttingen.

Cerri, Chiara 2011: Mut zur interkulturellen Literatur im DaF-Unterricht. In: Info DaF 38/4, S. 391-413.

Culler, Jonathan 2002: Literaturtheorie. Eine kurze Einführung. Aus dem Englischen übersetzt von Andreas Mahler. Stuttgart. Philipp Reclam jun. GmbH \& Co KG.

Dobstadt, Michael/Riedner, Renate 2017 (Hrsg.): Literatur Lesen Lernen. Lesewerkstatt Deutsch 2, von Nikolaus Euba und Chantelle Warner. Stuttgart: Klett.

العدد الخامس والعشرون (الجزء الرابع) 9 ا م r 
Ass.-Prof. Dr. Lamyaa Abdelmohsen Osman Ali Ziko

Dobstadt, Micheal/Riedner, Renate 2011: Fremdsprache Literatur - Neue Konzepte zur Arbeit mit Literatur im Fremdsprachenunterricht. In: Fremdsprache Deutsch 44, S. 5-14.

Ehlers, Swantje 1989: Sehen lernen. Zur ästhetischen Erfahrung im Kontext interkultureller Literaturvermittlung. In: Jahrbuch Deutsch als Fremdsprache 14, S. 171-197.

Ehlers, Swantje 2007: Lesen als Verstehen. Arbeit mit literarischen Texten. Fernstudieneinheit 2. Berlin; München; Wien; Zürich; New York: Langenscheidt.

Eismann, Volker/Thurmair, Maria 1993: Literatur für Lerner. Ein didaktisches Konzept für den Anfangsunterricht Deutsch als Fremdsprache. In: Jahrbuch Deutsch als Fremdsprache 19, S. 373389.

Esselborn, Karl 2010: Interkulturelle Literaturvermittlung zwischen didaktischer Theorie und Praxis. München: Iudiucium Verlag $\mathrm{GmbH}$.

GER 2001: Gemeinsamer europäischer Referenzrahmen für Sprachen: lernen, lehren, beurteilen. Berlin; München: Langenscheidt KG.

Helbig, Gerhard/Götze, Lutz/Henrici, Gert/Krumm, Hans-Jürgen 2001

(Hrsg.): Deutsch als Fremdsprache. Ein internationales Handbuch.

1. Halbband. Berlin; New York: Walter de Gruyter.

Honnef-Becker, Irmgard 1993: Wie sich Literatur mit Vergnügen lesen läßt. Bemerkungen zum Umgang mit literarischen Texten in Deutsch als Fremdsprache. In: Info Daf 20/4, S. 437-448.

Kayser, Wolfgang 1968: Das sprachliche Kunstwerk: eine Einführung in die Literaturwissenschaft. Bern: Francke Verlag.

Kramsch, Claire 2011: Symbolische Kompetenz durch literarische Texte. In: Fremdsprache Deutsch 44, S. 35-40.

Kramsch, Claire 2016: Symbolische Kompetenz im Zeitalter der Mehrsprachigkeit. Abrufbar unter: www.unibielefeld.de/lili/studium/faecher/daf/gastlehrstuhl-haraldweinrich/Claire_Kramsch_Rede_Harald-WeinrichGastlehrstuhl.pdf, 8pp. [Stand:18.4.2018].

Mecklenburg, Norbert 2003: Interkulturelle Literaturwissenschaft. In: Wierlacher/Bogner (Hrsg.), S. 433-439.

Rösch, Heidi 2011: Deutsch als Zweit- und Fremdsprache. Berlin: Akademie Verlag GmbH.

العدد الخامس والعشرون (الجزء الرابع) 9 ا م r

مجلة كلية التربية- جامعة عين شمس 


\section{Literatur im Rahmen akademischer Vorgaben der Germanistik-}

\section{Ausbildung: Ästhetische Impulse und didaktische Outcomes}

Rösch, Heidi 2016: Glokale Literatur im (Deutsch-als-) Fremdsprachunterricht. In: Info DaF 43/1, S. 3-20.

Schiedermair, Simone 2011: Literarische Texte als literarische Texte.

Vieldeutigkeit, Anschaulichkeit, Kontextverbundenheit. In:

Fremdsprache Deutsch 44, S. 28-34.

Schneider, Jost 1998: Einführung in die moderne Literaturwissenschaft. 2. durchgesehene Auflage. Bielefeld: Aisthesis Verlag.

Shalaby, Iman 2002/2003: Ästhetisch-kreative Entfaltung von Sinn. Zum

Stellenwert der Konkreten Poesie bei der Sensibilisierung ägyptischer Deutschlehrer-Studenten für deutschsprachliche Bedeutungsdifferenzierung. In: Kairoer Germanistische Studien 13, S. 247-296.

Tütken, Gisela 2006: Literatur im Unterricht Deutsch als Fremdsprache an der Hochschule im Ausland - aber wie? Ein Vorschlag am Beispiel Russlands: In: Info DaF 33/1, S. 52-90.

Ünal, Dalim Çigdem 2007: Lehr- und Lernprozesse fremdsprachlicher Literaturdidaktik: Eine Untersuchung mit türkischen DaFStudierenden am Beispiel des literarischen Rollenspiels. In: Zeitschrift für Interkulturellen Fremdsprachenunterricht 12:3, $23 \mathrm{~S}$. Abrufbar unter: https://tujournals.ulb.tu-darmstadt.de [Stand:18.4.2018]

Weinrich, Harald 1983: Literatur im Fremdsprachenunterricht - ja, aber mit Phantasie. In: Die neueren Sprachen, 82, S. 200-216.

Wierlacher, Alois/Bogner, Andrea 2003 (Hrsg.): Handbuch interkulturelle Germanistik. Stuttgart; Weimar: J. B. Metzler Verlag.

Zeuner, Ulrich 2002: Das Lehrmaterial „Einführung in die Landeskundedidaktik“. Von der Broschüre zum hybriden Lernarrangement. In: Info DaF 29/6, S. 530-549.

Ziko, Lamyaa A. O. A. 2012: Dantons Tod. Lektüre im literarischen DaFUnterricht im Rahmen der 25. Januar-Revolution von Ägypten. In: Zielsprache Deutsch 40/3, S. 19-33.

Ziko, Lamyaa A. O. A. 2013: Lyrik im universitären DaF-Unterricht in Ägypten. In: Faculty of Arts Journal. Edition No. 90, July 2013. AlMenoufia University, S. 3-24.

Ziko, Lamyaa A. O. A. 2019/2020: Interaktives Deutschlernen an ägyptischen Hochschulen: Traditionelle und digitale Möglichkeiten

العدد الخامس والعشرون (الجزء الرابع) 9 ا م ب

مجلة كلية التربية- جامعة عين شمس 
Ass.-Prof. Dr. Lamyaa Abdelmohsen Osman Ali Ziko

\begin{tabular}{l}
\hline im fremdsprachlichen Literaturunterricht. In: Riedner, \\
Renate/Schiedermair, Simone (Hrsg.): Literarisches und \\
alltäglisches Erzählen unter (fremd-)sprachendidaktischer \\
Perspektive. Iudicium Verlag (in Druck). \\
Ziko, Lamyaa A. O. A. 2019: Literatur, DaF und Kulturvermittlung im \\
universitären Kontext in Ägypten. In: Faculty of Languages \& \\
Translation's Journal. Scholarly International Refereed Journal, Al \\
Azhar University. No. 16, January 2019, 66-86.
\end{tabular}

\section{Arabische Literatur}

المعايير القومية الأكاديمية القياسية، قطاع كليات الآداب، 9 . . F

(NARS für Philosophische Fakultäten) Webseite abrufbar unter: www.du.edu.eg [Stand: 23.7.2017] 
Literatur im Rahmen akademischer Vorgaben der GermanistikAusbildung: Ästhetische Impulse und didaktische Outcomes 